\title{
Cortical Brain Activity is Influenced by Cadence in Cyclists
}

\author{
Kuno Hottenrott ${ }^{1}$, Marco Taubert ${ }^{2}$ and Thomas Gronwald ${ }^{1, *}$ \\ ${ }^{I}$ Department of Sport Science, Martin-Luther-University Halle-Wittenberg, Germany \\ ${ }^{2}$ Max Planck Institute for Human Cognitive and Brain Sciences, Leipzig, Germany
}

\begin{abstract}
The importance of the central nervous system in endurance exercise has not yet been exhaustively investigated because of difficulties in measuring cortical parameters in sport science. During exercise there are a lot of artifacts and perturbations which can affect signal quality of cortical brain activity. The technical developments of surface electroencephalography (EEG) minimize such influences during standardized test conditions on a bicycle ergometer. The aim of this study was to investigate how movement frequency affects cortical brain activity and established physiological parameters during exercise. In cycling peak performance is affected by cadence. The analysis of brain cortical activity might lead to new insights in the relation of power and cadence. In a laboratory study sixteen male, endurance-trained cyclists completed a $60 \mathrm{~min}$ endurance exercise on a high-performance bicycle ergometer. Cadence was changed every 10 min (90-120-60-120-60-90 rpm). EEG was used to analyze changes in cortical brain activity. Furthermore, heart rate, blood lactate and rate of perceived exertion (RPE) were measured after each cadence change. The results indicate that heart rate, blood lactate and RPE were higher at $120 \mathrm{rpm}$ compared to $60 \mathrm{rpm}$. The spectral EEG power increased statistically significantly in the alpha-2 and beta-2 frequency range by changing cadence from 60 to $120 \mathrm{rpm}$. By lowering the cadence from 120 to $60 \mathrm{rpm}$ the spectral power dropped statistically significantly in all analyzed EEG frequency bands. The data also showed a statistically significant decrease of spectral EEG power in all frequency ranges over time. In conclusion, the analyzed EEG data indicate that cadence should be considered as an independent exercise normative in the training process, because it directly influences metabolic, cardiac and cortical parameters.
\end{abstract}

Keywords: Brain waves, central nervous system, constant workload, eeg, movement frequency, pedaling frequency.

\section{INTRODUCTION}

Training loads cause complex reactions and stress different functional systems. Exercise-induced adaptations of the organism in the various organs and regulatory systems are already known, particularly of the cardiovascular and respiratory system as well as the energy metabolism. However, changes in brain activity during exercise have not been investigated sufficiently. This is due to difficulties in recording cortical brain activity during exercise. Recent studies indicate that technical developments of surface electroencephalography (EEG) led to new opportunities to examine brain activity during exercise [1-3]. These studies prove that artifact-free EEG signals can be recorded in motion under standardized conditions on a bicycle ergometer. In particular, the authors state that the influence of isolated exercise normatives on cortical brain activity has to be examined in future studies.

It has already been recognized that complexity and load of the training protocol are limited within training process, especially in cycling [4]. One of the largest reserves of training methodology is training based on stimulus change

*Address correspondence to this author at the Department of Sport Science, Martin-Luther-University Halle-Wittenberg, Von-Seckendorff-Platz 2, 06120 Halle (Saale), Germany; Tel: +49 (0) 34555244 29;

Fax: +49 (0) 34555270 54; E-mail: thomas.gronwald@sport.uni-halle.de and variability. Therefore, training at different cadences seems to be important to set new stimuli for the same power output in trained cyclists. For higher exercise economy, road cyclists should choose a low cadence of $60 \mathrm{rpm}$ [5-7]. However, in practice cyclists perform best at high $(90 \mathrm{rpm})$ to very high $(110 \mathrm{rpm})$ cadences [8]. Therefore, the best uphill performance is achieved with a higher cadence [9]. In track cycling an extremely high cadence enables the athlete to perform at his best [10], too. Despite the negative effects on the movement economy, a high cadence is necessary for a high power output in competitive sports. In this context, the analysis of brain cortical activity might lead to new insights in the relation of power and cadence.

On the one hand cardiorespiratory and metabolic responses to varying movement frequencies were adequately investigated [11-13], but on the other hand the effect of different cadences on the central nervous system has not been studied so far during exercise. Therefore, the purpose of this study was to examine the influence of different cycling cadences on cortical brain activity, heart rate, blood lactate and perception of effort.

\section{METHODS}

\section{Subjects}

Sixteen endurance-trained male cyclists were recruited from local cycling clubs (age: $25.9 \pm 3.8$ years; height: 180.7 
$\pm 6.1 \mathrm{~cm}$; body mass: $77.4 \pm 8.2 \mathrm{~kg}$; body fat: $12.3 \pm 3.4 \%$ ). Subjects had to be active cyclists with 10 to $12 \mathrm{~h}$ regular training or 350 to $450 \mathrm{~km}$ per week, non-smokers and righthanded. To check the dominant handedness, the translated version of the Edinburgh Handeness Inventory by Oldfield [14] was applied. Subjects were fully informed about the study design, possible risks and benefits. They all signed an informed consent form to participate in the study. A medical check-up was performed following the guidelines of the German Society for Sports Medicine and Prevention - DGSP [15]. Exclusion criteria were any cardiovascular, metabolic, neurological, pulmonary or orthopedic complications that could limit the performance of the exercise. It was made clear to the subjects that they were free to terminate the study at any time. The study was approved by the ethics committee of the medical faculty of the Martin-Luther-University Halle-Wittenberg.

\section{Test Protocol and Physiological Values}

Prior to testing, body mass and body fat were measured using a bio impedance device (Tanita, model BC-545 InnerScan, Germany). The performance of the cyclists was assessed in a cardiopulmonary exercise stage test (start: 100 $\mathrm{W}$, increment: $20 \mathrm{~W}$, length: $3 \mathrm{~min}$ ) with a spirometry system (Cortex, Metamax 3b, Germany) on a highperformance bicycle ergometer (FES, E 2000s, Germany). The stage test was carried out until voluntary exhaustion (Respiratory quotient $>1.1$ or the inability to keep up the power output). Maximal load $\left(\mathrm{P}_{\max }\right)$ and peak oxygen consumption $\left(\mathrm{VO}_{2 \text { peak }}\right)$ were determined at the end of the test. After each stage blood lactate were measured with the enzymatic-amperometric method (Dr. Müller, Super GL ambulance, Germany) in $10 \mu \mathrm{l}$ blood taken from an ear lobe. According to Dickhuth et al. [16], the aerobic lactate threshold, defined as the lowest value of the quotient lactate and power $\left[\left(\mathrm{mmoll}^{-1}\right) \cdot(\text { watt })^{-1}\right]$, and the anaerobic lactate threshold were identified. Collected data were analyzed with WinLactat 3.1 (Mesics GmbH, Germany).

One week later subjects performed a 60 min exercise test with varying cadences at a power of $90 \%$ of anaerobic lactate threshold. During the exercise test, the subjects took an individual driving and seat position. Subjects were instructed to hold their torso and head as steady as possible during pedaling. There was a standardized warm up (10 min at $100 \mathrm{~W}, 5 \mathrm{~min}$ at $150 \mathrm{~W}$ ) prior to the test and a cool down $(10 \mathrm{~min}$ at $100 \mathrm{~W})$ afterwards. During the exercise pedal frequency was changed (90-120-60-120-60-90 rpm) every 10 min. Heart rate values were measured and recorded in 5-s intervals with Polar S810i and Polar Wearlink Coded (Polar Electro $\mathrm{GmbH}$, Germany) and the rate of perceived exertion (RPE) was assessed using the BORG-scale [17]. Blood lactate was measured as described in the stage test. Heart rate, blood lactate and RPE were assessed at rest, after warm up, before every cadence change and after cool down.

\section{EEG Recordings and Analysis}

For continuous recording of brain cortical activity electroencephalography (QuickAmp-EEG-system 72-channel, Brain Products, Germany) was used. The changes in brain wave signals were recorded with 32 active surface electrodes (Brain Products, actiCAP, Germany; Fp1, Fp2, F7, F3, Fz, F4, F8, FC5, FC1, FC2, FC8, T7, C3, Cz, C4, T8, TP9, CP5, CP1,
CP2, CP6, TP10, P7, P3, Pz, P4, P8, PO9, O1, Oz, O2, PO10) fixed in an flexible EEG cap (EasyCAP, Germany), aligned to the international 10:20 system [18]. The cap was suitable for various head sizes, breathable to prevent heat accumulation and fixed with a chin strap. Data were measured continuously with open eyes. The active $\mathrm{Ag} / \mathrm{AgCl}$ electrodes used in the system have implemented active circuitry (actiShield) that stabilize the signal and allow recordings of high contact resistance up to $60 \mathrm{k} \Omega$. Consequently, this active shielding technology and the high input impedance of the amplifier made it possible to suppress artifacts due to external electrical influences as well as cable movement. This also led to an optimal signal-noise ratio. The subject's head was equipped with the cap and electrodes were filled with a high viscous electrolyte gel (SUPER-VISC, EasyCAP, Germany) to receive an optimal connection to the scalp (impedances: $<20 \mathrm{k} \Omega$ ) 40 min before the investigation. The electrode impedances were controlled with the actiCAP Software 1.2 (Brain Products, Germany) and the signal was recorded with Brain Vision Recorder 1.03 (Brain Products, Germany) software. Data were sampled at $500 \mathrm{~Hz}$. To avoid perspiration artifacts with cross connection between electrodes a ventilator was used to cool the breathable EEG cap.

The Brain Vision Analyzer 2.0 (Brain Products, Germany) software was used for off-line processing of spontaneous EEG raw data. EEG data were analyzed at rest, after warm up, before every cadence change, after cool down and at rest following the exercise. After editing the markers, data were filtered (high-pass: $1.5 \mathrm{~Hz}$, slope: $12 \mathrm{~dB}^{-1}$ octave $^{-1}$; low-pass: $40 \mathrm{~Hz}$, Slope: $48 \mathrm{~dB}^{-1} \operatorname{cotave}^{-1}$ ) to remove movement and muscle artifacts, which were expected at 0.5 $1.5 \mathrm{~Hz}$ and $>40 \mathrm{~Hz}$ [19]. Artifacts up to theta-frequencies $(4.5 \mathrm{~Hz})$ and down to beta-2-frequencies $(32 \mathrm{~Hz})$ were excluded by the use of frequency-analysis. Afterwards, a manual raw data inspection was carried out to highlight visually identified artifacts. Independent Component Analysis (ICA) was subsequently calculated to remove artifacts from the signal [20]. This off-line control made sure that only artifact-free EEG segments were included into the analysis. Based on the edited markers, signals were segmented into 4s-data-sets with a corrected baseline [21] in the last minute of each measuring time point. Of every subject and measurement time point five artifact-free segments were analyzed and averaged using a Fast-FourierTransformation (maximum resolution, power in $\mu \mathrm{V}^{2}$, use of full spectrum, Hanning window, window length: $20 \%$ ). The received frequency spectrum was divided into five frequency bands (theta: $4.5-7.5 \mathrm{~Hz}$, alpha-1: 7.5-10 Hz, alpha-2: 10$12.5 \mathrm{~Hz}$, beta-1: $12.5-18 \mathrm{~Hz}$, beta-2: $18-32 \mathrm{~Hz}$ ) and described by the absolute spectral power $\left[\mu \mathrm{V}^{2}\right]$. For further processing, the values were exported to Microsoft Excel 2007. The analysis was performed for all 32 electrodes per frequency range and measurement time point. The absolute values of spectral power were calculated as percentage change from the individual rest condition $(100 \%)$ to exclude the influence of different EEG basic types [2]. Data from TP9, PO9, O1, Oz, O2, PO10 and TP10 were completely excluded because of muscle artifacts in the neck area.

\section{Statistical Analysis}

All data were analyzed with the software SPSS Statistics 17.0. To evaluate whether or not the data were normally 
Table 1. Heart Rate, Blood Lactate and RPE during Warm Up, During Constant Workload and Exposure to Different Cadences and During Cool Down. Results are Expressed as Mean $\pm s$

\begin{tabular}{|c|c|c|c|c|}
\hline & $\begin{array}{c}\text { Cadence } \\
\text { [rpm] }\end{array}$ & $\begin{array}{c}\text { Heart Rate } \\
{\left[\mathrm{min}^{-1}\right]}\end{array}$ & $\begin{array}{l}\text { Blood Lactate } \\
{\left[\mathrm{mmoll}^{-1}\right]}\end{array}$ & $\begin{array}{c}\text { RPE } \\
{[6-20]}\end{array}$ \\
\hline Rest & - & $62.56 \pm 8.74$ & $1.01 \pm 0.34$ & - \\
\hline Warm up & 90 & $118.50 \pm 8.61 * *$ & $0.80 \pm 0.40 * *$ & $9.25 \pm 1.91$ \\
\hline \multirow{6}{*}{$\begin{array}{c}\text { Constant workload at } \\
90 \% \text { of the } \mathrm{LT}\end{array}$} & 90 & $144.13 \pm 9.69^{* *}$ & $1.51 \pm 0.58^{* *}$ & $13.06 \pm 1.12 * *$ \\
\hline & 120 & $163.81 \pm 9.84^{* *}$ & $3.45 \pm 1.10 * *$ & $15.81 \pm 1.17^{* *}$ \\
\hline & 60 & $150.25 \pm 10.47^{* *}$ & $1.71 \pm 0.57 * *$ & $13.75 \pm 1.48^{* *}$ \\
\hline & 120 & $168.81 \pm 10.75^{* *}$ & $3.49 \pm 1.17^{* *}$ & $16.50 \pm 1.79 * *$ \\
\hline & 60 & $154.88 \pm 10.67 * *$ & $1.82 \pm 0.45^{* *}$ & $14.31 \pm 1.58^{* *}$ \\
\hline & 90 & $157.50 \pm 11.12 * *$ & $1.74 \pm 0.58$ & $14.25 \pm 1.39$ \\
\hline Cool down & 90 & $118.69 \pm 8.55^{* *}$ & $1.00 \pm 0.23^{* *}$ & $8.31 \pm 1.85^{* *}$ \\
\hline
\end{tabular}

$\mathrm{p} \leq 0.01 * *$ to previous measurement time point.

Table 2. Percentage Change in the Spectral EEG Power in all Frequency Ranges at Rest (100\%), During Warm Up, During Constant Workload and Exposure to Different Cadences, During Cool Down and Following Exercise. Results are Expressed as Mean $\pm s$

\begin{tabular}{|c|c|c|c|c|c|c|}
\hline & $\begin{array}{c}\text { Cadence } \\
\text { [rpm] }\end{array}$ & $\begin{array}{c}\text { Theta } \\
\text { [\%] }\end{array}$ & $\begin{array}{c}\text { Alpha-1 } \\
{[\%]}\end{array}$ & $\begin{array}{c}\text { Alpha-2 } \\
{[\%]}\end{array}$ & $\begin{array}{c}\text { Beta-1 } \\
{[\%]}\end{array}$ & $\begin{array}{c}\text { Beta-2 } \\
{[\%]}\end{array}$ \\
\hline Rest & - & 100 & 100 & 100 & 100 & 100 \\
\hline Warm up & 90 & $351 \pm 159^{* *}$ & $282 \pm 200^{* *}$ & $250 \pm 116^{* *}$ & $231 \pm 129^{* *}$ & $257 \pm 170^{* *}$ \\
\hline \multirow{6}{*}{$\begin{array}{c}\text { Constant workload at } \\
90 \% \text { of the } \mathrm{LT}\end{array}$} & 90 & $347 \pm 191$ & $308 \pm 284$ & $273 \pm 188$ & $229 \pm 111$ & $223 \pm 133$ \\
\hline & 120 & $317 \pm 200$ & $361 \pm 395$ & $319 \pm 296$ & $256 \pm 146$ & $225 \pm 141$ \\
\hline & 60 & $203 \pm 133^{*}$ & $171 \pm 112^{*}$ & $165 \pm 123^{*}$ & $144 \pm 83^{* *}$ & $142 \pm 109^{* *}$ \\
\hline & 120 & $259 \pm 176$ & $334 \pm 455$ & $341 \pm 395^{*}$ & $209 \pm 165$ & $197 \pm 166^{*}$ \\
\hline & 60 & $166 \pm 90^{*}$ & $143 \pm 80$ & $136 \pm 82 *$ & $111 \pm 57^{* *}$ & $111 \pm 77 * *$ \\
\hline & 90 & $171 \pm 84^{*}$ & $152 \pm 83$ & $154 \pm 112$ & $152 \pm 109^{*}$ & $144 \pm 141$ \\
\hline Cool down & 90 & $147 \pm 116^{*}$ & $114 \pm 78$ & $106 \pm 101^{*}$ & $103 \pm 74 * *$ & $112 \pm 107^{*}$ \\
\hline Rest & - & $37 \pm 22 * *$ & $41 \pm 22 * *$ & $41 \pm 21 *$ & $38 \pm 21 * *$ & $36 \pm 21^{*}$ \\
\hline
\end{tabular}

$\mathrm{p} \leq 0.05^{*}, \mathrm{p} \leq 0.01^{* *}$ to previous measurement time point.

distributed the Kolmogorov-Smirnov test was applied. In case of normal distribution, differences between measurement time points were calculated (spectral EEG power $[\%]$, heart rate $\left[\mathrm{min}^{-1}\right]$, blood lactate $\left.\left[\mathrm{mmoll}^{-1}\right]\right)$ using ANOVA for repeated measures and student's t-test for paired samples. For ordinal data (RPE) the Wilcoxon test was used. The different levels of statistical significance were set at $\mathrm{p} \leq 0.05(*)$ and $\mathrm{p} \leq 0.01(* *)$.

\section{RESULTS}

Subjects achieved a mean $\mathrm{P}_{\max }$ of $338.33 \pm 30.75 \mathrm{~W}(90$ $\%$ of anaerobic lactate threshold: $236.36 \pm 24.49 \mathrm{~W})$ and $\mathrm{VO}_{2 \text { peak }}$ of $54.03 \pm 6.11 \mathrm{ml} \cdot \mathrm{min}^{-1} \cdot \mathrm{kg}^{-1}$ in the cycle ergometer stage test. All sixteen subjects completed the study.

The values heart rate and blood lactate increased statistically significantly $(\mathrm{p} \leq 0.01)$ during the warm up period and at the beginning of the exercise test compared to the rest condition. The rate of perceived exertion increased statistically significantly $(\mathrm{p} \leq 0.01)$ at the beginning of the exercise test compared to the warm up period. At $120 \mathrm{rpm}$ heart rate, blood lactate and RPE were significantly higher ( $\mathrm{p}$ $\leq 0.01$ ) than at a cadence of $60 \mathrm{rpm}$. All physiological values decreased statistically significantly $(p \leq 0.01)$ during the cool down period, see Table 1.

The spectral EEG power increased statistically significantly $(\mathrm{p} \leq 0.01)$ in all analyzed frequency ranges from rest condition to the warm up (theta: $+251 \%$, alpha-1: $+182 \%$, alpha-2: $+149 \%$; beta- $1:+131 \%$, beta- $2:+157 \%$ ). During exercise the spectral EEG power was in almost all frequency ranges significantly higher at $120 \mathrm{rpm}$ compared to $60 \mathrm{rpm}$. The cadence changes are reflected in the EEG spectral power, see Table 2, Fig. (1) and Fig. (2). By comparing the measurement time points under identical conditions at the beginning and the end of the constant workload and $90 \mathrm{rpm}$ cadence, a statistically significant 


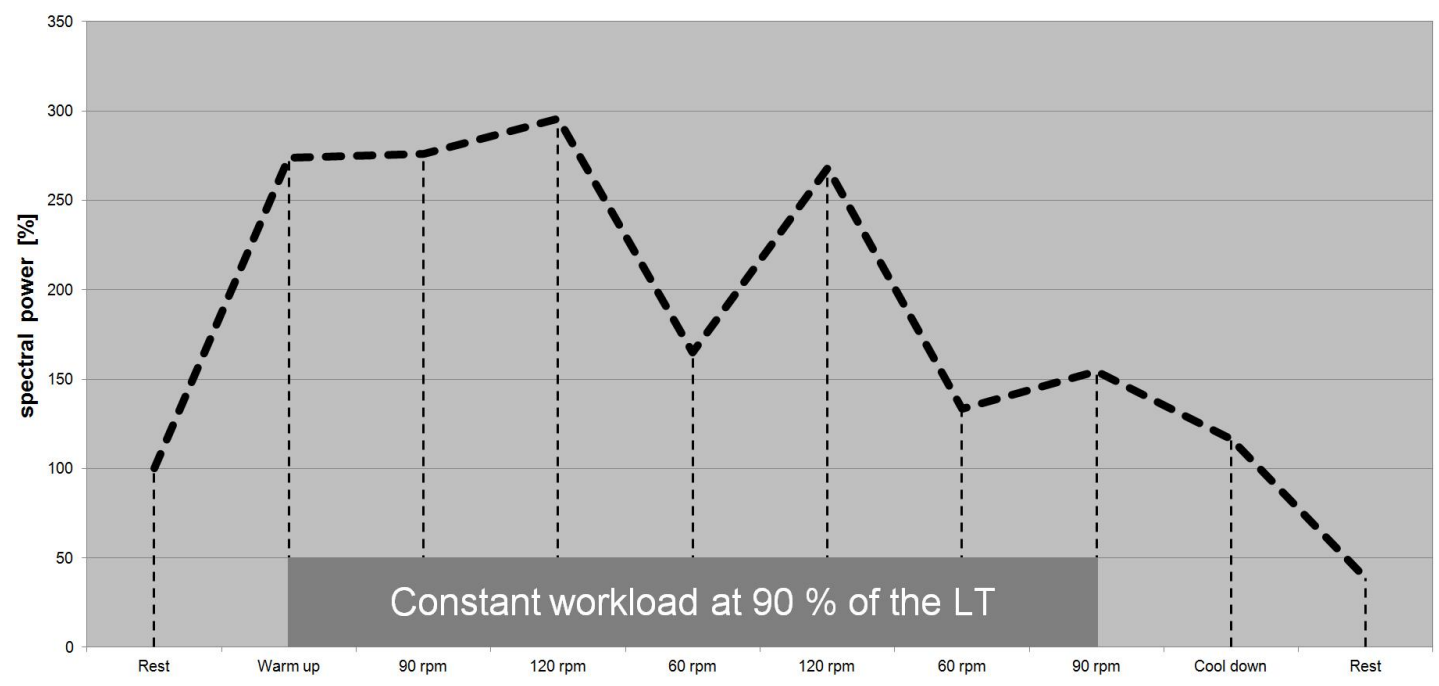

Fig. (1). Percentage change in spectral power calculated over 32 electrodes compared to the rest condition (100\%) and averaged over the entire EEG frequency range $(4.5-32 \mathrm{~Hz})$ and all subjects.

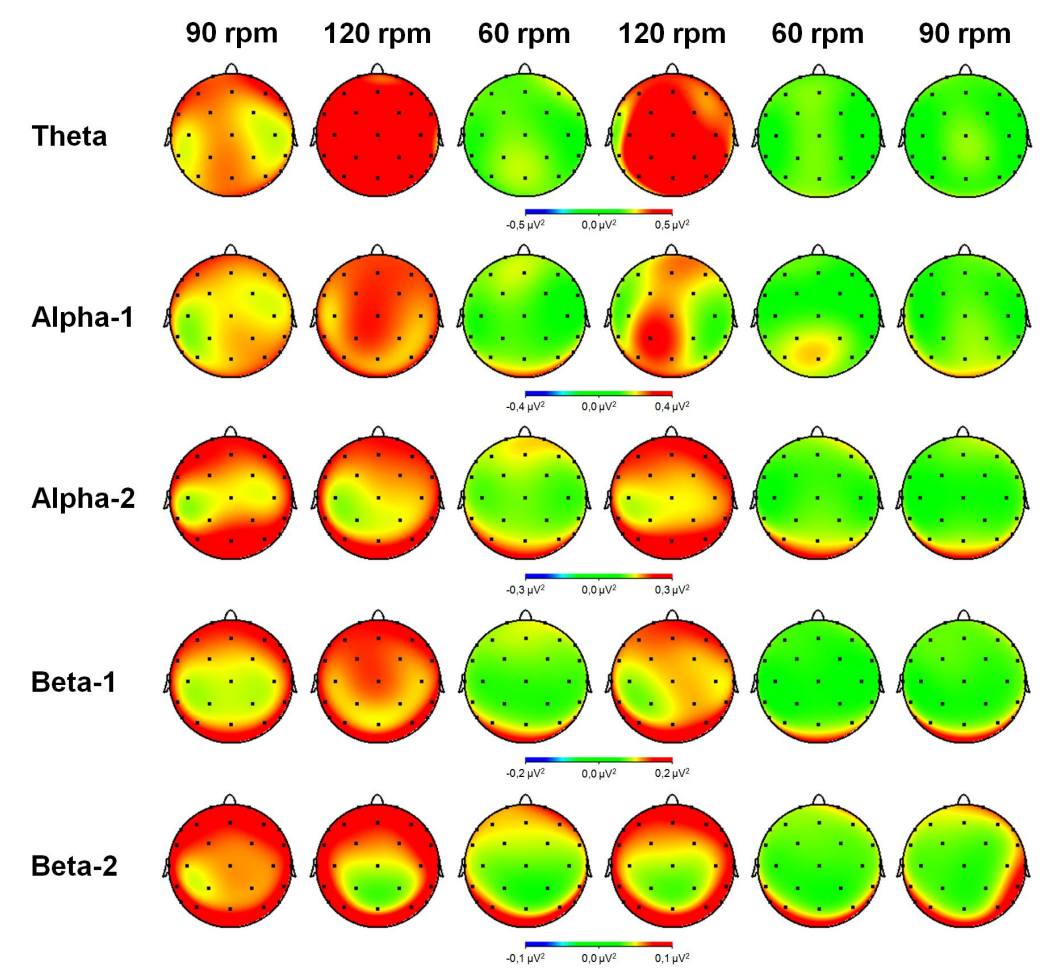

Fig. (2). Mapping of all EEG frequency ranges; higher values of the spectral power (red) by increasing cadence to 120 rpm and lowering of the spectral power (green) after lowering the cadence to $60 \mathrm{rpm}$. Lower spectral EEG power at the end of the constant workload (90 rpm) compared to the beginning $(90 \mathrm{rpm})$.

decrease in EEG power was measured in the theta, alpha-2, beta-1, beta-2 $(\mathrm{p} \leq 0.01)$ and in the alpha-1 $(\mathrm{p} \leq 0.05)$ frequency range (theta: $-176 \%$, alpha-1: $-156 \%$, alpha-2: $-119 \%$, beta-1: $-77 \%$, beta-2: $-79 \%$ ). The cortical activity at rest decreased statistically significantly $(p \leq 0.01)$ in all frequency ranges from the initial level before exercise to the resting state after exercise (theta: $-63 \%$, alpha-1: $-59 \%$, alpha-2: $-59 \%$, beta-1: $-62 \%$, beta-2: $-64 \%$ ).

\section{DISCUSSION}

At the same power output the heart rate increased at a higher cadence $(120 \mathrm{rpm})$. These findings are consistent with those of other studies [11, 12, 22, 23]. Despite decreasing pedal forces the need to stabilize the upper body and the trunk area more at higher frequencies results in increased cardiac stress [24]. Consequently, the recruitment of additional muscle fibres from the peripheral body, increased inter-muscular coordination and a higher cardiovascular stress is necessary to maintain higher cadences $[25,26]$. Kromer et al. [25] also found an increased heart rate at a higher crank rate in hand cycling.

Blood lactate increased at a high cadence $(120 \mathrm{rpm})$ and decreased at a low cadence $(60 \mathrm{rpm})$. These results confirm the influence of the cadence on the energy supply, which 
was also found in previous studies [11-13, 27]. Furthermore, it is already known that blood lactate increases with higher cadences and work load [28, 29]. Given a constant work load, the blood lactate increases more at intensities higher than 200 watts and cadences from 100 to $120 \mathrm{rpm}[12,13$, 26]. The change in blood lactate at different cadences is mainly due to the activation and recruitment of different muscle fibre types [30, 31]. These facts depend on the activation of fast-twitch muscle fibres in the exercise program [32]. Due to a higher heart rate with increasing cadence, some authors also state that a stronger and more efficient blood flow is elicited in stressed muscles, which causes an increased washout of lactate in the blood [11, 23, $25]$.

The increased perception of effort at a higher cadence is related with the increase in heart rate and blood lactate concentration. Consequently, the increased cardiac and metabolic stress results in a higher perception of effort [22, 23, 33, 34]. Furthermore, Bailey et al. [35] confirmed a correlation between perception of effort and EEG spectral power. The most significant relation was found between RPE and changes in delta frequency range.

The main purpose of the present study was to characterize brain cortical activity at different cadences. The spectral EEG power in all frequency ranges depended on the cadence while the workload was held constant. Brümmer et al. [36] indicate a relation between central activation, exercise mode and intensity. However, the influence of different cycling cadences on brain cortical activity has not been investigated before. The characteristics described previously could be due to the increased psychophysiological stress at $120 \mathrm{rpm}$.

During and immediately after the warm up the highest increase in EEG power was found. These findings confirm the results of Brümmer et al. [21], who recorded EEG during an incremental exercise test on a bicycle ergometer. This can be interpreted as acute demand on the whole organism and the central nervous regulatory mechanisms. High cortical activation is necessary to provide high performance and power output in sports. The statistically significant decrease of cortical brain activity in all frequency ranges over time may indicate fatigue. Baumeister et al. [37] also showed a decrease in cortical brain activation after a prolonged exhaustive exercise protocol in theta and alpha frequencies. Reinecke et al. [38] demonstrated this tendency in the beta frequency range under similar conditions.

In this context gained data can be interpreted in terms of the central governor model of Noakes [39]. This model postulated a central nervous system regulatory mechanism, which controls physical activity according to all inputs and outputs of the human organism to protect the homeostasis. This control mechanism does not restrict to the function of the heart or skeletal muscle, but it regulates the power output by controlling the number of recruited muscle fibres or motor units involved in the working muscles [39]. Therefore, all sensory information from the body periphery is processed to regulate the degree of activation. The cortical brain activation decreases with the onset of fatigue because of the reduced activation impulses to the working muscles. As already hypothesized by Beyer and Schumann [40], the curve of the cortical brain activity over time resembled and resulted in an inverted U-shape.

\section{LIMITATIONS}

As EEG was recorded during exercise physiological and mechanical parameters might have affected the electronical signal. The methodological approach, which was in line with previous investigators [2], was designed to reduce the potential impact of these factors. Subjects were instructed to hold their torso and head as steady as possible during pedaling. Additionally, recordings from occipital and temporal regions (TP9, PO9, O1, Oz, O2, PO10, TP10) were excluded from the analysis because of muscle artifacts. The electronical signals from all remaining electrodes were averaged for every frequency range and measurement time. The interpretation of data was limited, because investigators could not pay attention to regional differences and the source of the signal. Furthermore, it remains unclear to which extent peripheral physiology accounted for changes in EEG signal.

\section{CONCLUSION}

The results of the present study indicate that cortical brain activation can be measured during cycling exercise under laboratory conditions. EEG recordings at different cadences but identical workload showed that higher pedal frequencies are associated with an increase in brain cortical activity, heart rate, blood lactate and RPE. The U-shaped curve of EEG spectral power over time suggests that central activation decreases with the onset of fatigue. Consequently, maintaining a high level of brain cortical activation might be necessary to delay fatigue and improve racing performance.

Training at different cadences seems to be the key to respond variably to different requirements during a race (e.g. short burst or final sprint). The complexity and repertoire of suitable control programs in the central nervous system could be extended with specific cadence training. To increase the power output at higher cadences, higher cortical brain activation is necessary. Increased cortical brain activation at higher pedal frequencies could possibly be maintained longer by performing a systematic training at different cadences. Coaches and athletes should therefore include variable systematic training sessions at low cadences and high cadences to improve performance in training and competition. Future studies should focus on evaluating specific training programs that potentially increase central activation during exercise.

\section{CONFLICT OF INTEREST}

The authors confirm that this article content has no conflicts of interest.

\section{ACKNOWLEDGEMENTS}

None declared.

\section{REFERENCES}

[1] Hall EE, Martin BE, Bailey SP, Miller PC, Folger SE. Changes in EEG activity during exercise: Due to Duration or intensity of exercise? Med Sci Sports Exerc 2006; 38(5) (Suppl): 54.

[2] Bailey SP, Hall EE, Folger SE, Miller PC. Changes in EEG during graded exercise on a recumbent cycle ergometer. J Sports Sci Med 2008; 4(7): 505-11. 
[3] Fumoto M, Oshima T, Kamiya K, et al. Ventral prefrontalcortex and serotonergic system activation during pedalling exercise induces negative mood improvement and increased alpha band in EEG. Behav Brain Res 2010;213(1): 1-9.

[4] Faria EW, Parker DL, Faria IE. The science of cycling. Physiology and training - Part 1. Sports Med 2005; 35(4): 285-312.

[5] Marsh AP. What determines the optimal cadence. Cycling Sci 1996; 1: 1-12.

[6] Hansen EA, Smith G. Factors affecting cadence choice during submaximal cycling and cadence influence on performance. Int $\mathbf{J}$ Sports Physiol Perform 2009; 4(1): 3-17.

[7] Vercruyssen F, Brisswalter J. Which factors determine the freely chosen cadence during submaximal cycling? J Sci Med Sport 2010; 13(2): 225-31.

[8] Faria EW, Parker DL, Faria IE. The science of cycling. Factors affecting performance - Part 2. Sports Med 2005; 35(4): 313-37.

[9] Neumann G. Physiologische Grundlagen des Radsports. Dtsch Z Sportmed 2000; 51(5): 169-75.

[10] Emanuele U, Denoth J. Radfahren: Trittfrequenz und Tritttechnik in der Ebene und am Berg. Schweiz Z Med Traumatol 2008; 56(2): 71-6.

[11] Gotshall RW, Bauer TA, Fahmer SL. Cycling cadence alters exercise hemodynamics. Int J Sports Med 1996; 17(1): 17-21.

[12] Chavarren J, Calbet JAL. Cycling efficiency and pedaling frequency in road cyclists. Eur J Appl Physiol Occup Physiol 1999; 80(6): 555-63.

[13] Brisswalter J, Hausswirth C, Smit D, Vercruyssen F, Vallier, JM. Energetically optimal cadence vs. Freely-chosen cadence during cycling: Effect of exercise duration. Int J Sports Med 2000; 21(1): 60-4.

[14] Oldfield RC. The assessment and analysis of handedness: The Edinburgh inventory. Neuropsychologia 1971; 9(1): 97-13.

[15] Deutsche Gesellschaft für Sportmedizin und Prävention - DGSP. S1-Leitlinie Vorsorgeuntersuchung im Sport. 2007 [cited 2013 February 10]. Available from: http://www.dgsp.de/_downloads/allgemein/S1_Leitlinie.pdf

[16] Dickhuth $\mathrm{H}-\overline{\mathrm{H}}$, Aufenanger W, Rokitzki L, Huonker M, Keul J. Lactate performance curve, performance assessment and training management of high performance athletes in middle- and longdistance running. Int J Sports Med 1988; 9: 387.

[17] Borg G. Borg's perceived exertion and pain scales. Champaign: Human Kinetics 1998.

[18] Jasper HH. The ten twenty electrode system of the International Federation. Electroencephalogr Clin Neurophysiol 1958; 10: 371-5.

[19] Hilty L, Langer N, Pascual-Marqui R Boutellier U, Lutz K. Fatigue-induced increase in intracortical communication between $\mathrm{mid} /$ anterior insular and motor cortex during cycling exercise. Eur J Neurosci 2011; 34(12): 2035-42.

[20] Jung T-P, Makeig S, Bell AJ, Sejnowski TJ. Independent component analysis of electroencephalographic and eventrelated potential data. In: Poon P, Brugge J, Eds. Auditory Processing and Neural Modeling. New York: Plenum Press 1998; pp. 189-97.

[21] Brümmer V, Schneider S, Strüder HK, Askew CD. Primary motor cortex activity is elevated with incremental exercise intensity. Neuroscience 2011b; 181(1): 150-62.

[22] Löllgen H, Graham T, Sjogaard G. Muscle metabolites, force, and perceived exertion bicycling at varying pedal rates. Med Sci Sports Exerc 1980; 12(5): 345-51.
[23] Hagberg JM, Mullin JP, Giese MD, Spitznagel E. Effect of pedaling rate on submaximal exercise responses of competitive cyclists. J Appl Physiol 1981; 51(2): 447-51.

[24] Sargeant AJ. Human power output and muscle fatigue. Int J Sports Med 1994; 15(3): 116-21.

[25] Kromer P, Hirschmüller A, Dickhuth H-H, Gollhofer A, Röcker K. Der Einfluss der Kurbelfrequenz im Handcycling auf unterschiedliche Referenzpunkte der Laktatleistungskurve. Dtsch Z Sportmed 2011; 62(1): 22-8.

[26] Foss $\varnothing$, Hallén J. The most economical cadence increases with increasing workload. Eur J Appl Physiol 2004; 92(4/5): 443-51.

[27] Mc Naughton L, Thomas D. Effects of differing pedaling speeds on the power-duration relationship of high intensity cycle ergometry. Int J Sports Med 1996; 17(4): 287-92.

[28] Buchanan M, Weltman A. Effects of pedal frequency on $\mathrm{VO}_{2}$ and work output at lactate threshold (LT), fixed blood lactate concentrations of $2 \mathrm{mM}$ and $4 \mathrm{mM}$, and $\max$ in competitive cyclists. Int J Sports Med 1985; 6(3): 163-8.

[29] Mora-Rodriguez R, Aguado-Jimenez R. Performance at high pedaling cadences in well-trained cyclists. Med Sci Sports Exerc 2006; 38(5): 953-7.

[30] Patterson RP, Moreno MI. Bicycle pedaling forces as a function of pedaling rate and power output. Med Sci Sports Exerc 1990; 22(4): 512-6.

[31] Ahlquist LE, Bassett DR, Sufit R, Nagle FJ, Thomas DP. The effect of pedaling frequency on glycogen depletion rates in type I and type II quadriceps muscle fibres during submaximal cycling exercise. Eur J Appl Physiol 1992; 65(4): 360-4.

[32] Hollmann W, Strüder HK. Sportmedizin. Grundlagen für körperliche Aktivität, Training und Präventivmedizin. Stuttgart: Schattauer 2009

[33] Stamford BA, Noble BJ. Metabolic cost and perception of effort during bicycle ergometer work performance. Med Sci Sports Exerc 1974; 6(4): 226-31.

[34] Marsh AP, Martin PE. The association between cycling experience and preferred and most economical cycling cadences. Med Sci Sports Exerc 1993; 25(11): 1269-74.

[35] Bailey, SP, Hall, EE, Miller, PC, Folger, SE. Changes in brain activity, affect, and perception during grades exercise. Med Sci Sports Exerc 2005; 37(5): S207.

[36] Brümmer V, Schneider S, Abel T, Vogt T, Strüder HK. Brain cortical activity is influenced by exercise mode and intensity. Med Sci Sports Exerc 2011a; 43(10): 1863-72.

[37] Baumeister J, Reinecke K, Schubert M, Schade J, Weiss M. Effects of induced fatigue on brain activity during sensorimotor control. Eur J Appl Physiol 2012; 112(7): 2475-82.

[38] Reinecke K, Schubert M, Weiß M, Baumeister J. Divergente kortikale Aktivierung nach erschöpfendem Kraft- und Ausdauertraining: eine EEG-Studie. Dtsch Z Sportmed 2012; 63(78): 250.

[39] Noakes TD. Time to move beyond a brainless exercise physiology: The evidence for complex regulation of human exercise performance. Appl Physiol Nutr Metab 2011; 36(1): 23-35.

[40] Beyer L, Schumann H. Möglichkeiten neurophysiologischer Untersuchungen in der Sportmedizin anhand von zwei ausgewählten Beispielen. Med Sport 1981; 21: 65-70.

This is an open access article licensed under the terms of the Creative Commons Attribution Non-Commercial License (http://creativecommons.org/licenses/ by-nc/3.0/) which permits unrestricted, non-commercial use, distribution and reproduction in any medium, provided the work is properly cited. 